\title{
Selección del tiempo de retardo en el gráfico de Poincaré: Medición de los niveles de sedación y analgesia con señales EEG
}

\author{
Time-Delay selection in Poincaré plot: assessing \\ sedation-analgesia levels with EEG signals
}

\author{
Jose Daniel Bolaños $^{1} \quad$ Jose Fernando Valencia $^{1 *} \quad$ Montserrat Vallverdú $^{2}$ \\ Daniel Felipe Valencia ${ }^{1} \quad$ Xavi Borrat $^{3} \quad$ Pedro Luis Gambús ${ }^{3}$ \\ Recibido 4 de octubre de 2018, aceptado 17 de diciembre de 2019 \\ Received: October 4, 2018 Accepted: December 17, 2019
}

\begin{abstract}
RESUMEN
El gráfico de Poincaré es un método no lineal que permite analizar la variabilidad de una señal, dibujando dicha señal contra ella misma retardada en el tiempo. Aunque este método ha sido aplicado al análisis del electroencefalograma (EEG) para la medición de niveles de anestesia general, su aplicación en la medición de niveles de anestesia ligera aún no se ha abordado con profundidad. En este trabajo, se consideran varios tiempos de retardo en el gráfico de Poincaré para caracterizar el nivel de anestesia ligera en 110 pacientes sometidos a endoscopia digestiva. Los índices de Poincaré son combinados con parámetros espectrales de señales que contienen información del EEG y del EMG (electromiografía) para modelar los niveles de anestesia ligera, teniendo como referencia la escala de sedación Ramsay. La mejor predicción, utilizando índices del gráfico de Poincaré, del nivel de sedación y analgesia $(\mathrm{Pk}=0,711)$ se obtiene usando un retardo de $8 \mathrm{~ms}$ en el rango de frecuencia de 30-110 Hz y de $6 \mathrm{~ms}$ en el rango de frecuencia de $0,5-30 \mathrm{~Hz}$, respectivamente. La combinación de diferentes parámetros permitió obtener valores de $\mathrm{Pk}=0,799$.
\end{abstract}

Palabras clave: Análisis espectral, electroencefalograma, gráfico de Poincaré, sedación-analgesia, tiempo de retardo.

\begin{abstract}
Poincare plot is a non-linear method which allows analyzing the variability of a signal by plotting the signal against itself after a delay time. Although this method has been used to analyze the electroencephalogram (EEG) in order to assess general anesthesia, its application on light sedation has not been addressed in depth yet. In this work, several time-delays are considered in the Poincaré plot to characterize levels of light anesthesia on 110 patients scheduled to digestive endoscopy. Poincaré Plot indexes are mixed with parameters obtained from spectral analysis of signals with EEG and EMG (electromyography) information in order to model light anesthesia levels, according to Ramsay Sedation Scale. The best prediction, using indexes from Poincaré plot, of sedation level and analgesia level $(P k=0.711)$ is obtained with a time delay of $8 \mathrm{~ms}$ in a frequency range from $30 \mathrm{~Hz}$ to $110 \mathrm{~Hz}$ and $6 \mathrm{~ms}$ in a frequency range from $0.5 \mathrm{~Hz}$ to $30 \mathrm{~Hz}$, respectively. $P k=0.799$ was obtained with a model mixing different parameters.
\end{abstract}

Keywords: Delay time, electroencephalogram, Poincaré plot, sedation-analgesia, spectral analysis.

1 Universidad de San Buenaventura. Programa de Ingeniería Electrónica. Cali, Colombia.

E-mail: jdbolanos@usbcali.edu.co; jfvalenc@usbcali.edu.co; dfvalencia1@usbcali.edu.co

2 Universitat Politècnica de Catalunya - BarcelonaTech (UPC). Departamento Automática, Centro de Investigación en Ingeniería Biomédica. Barcelona, España. E-mail: montserrat.vallverdu@upc.edu

3 Hospital CLINIC de Barcelona. Departamento de Anestesia. Barcelona, España.

E-mail: xborrat@hospitalclinic.org; plgambus@hospitalclinic.org

* Autor de correspondencia: jfvalenc@usbcali.edu.co 


\section{INTRODUCCIÓN}

La sedación-analgesia es un proceso continuo inducido por medicamentos que comprende cuatro niveles, desde una sedación mínima (ansiólisis) hasta la anestesia general [1]. En el primer nivel las funciones respiratorias y cardiovasculares no se ven afectadas y los pacientes pueden responder normalmente a los comandos verbales, aunque la función cognitiva y la coordinación pueden verse afectadas. En el segundo nivel la ventilación es adecuada y la función cardiovascular generalmente se mantiene, evitando cualquier intervención para mantener una vía aérea abierta. Los pacientes pueden responder a propósito a los comandos verbales, ya sea solos o acompañados por una ligera estimulación táctil. En el tercer nivel generalmente se mantiene la función cardiovascular, pero la capacidad para mantener la función de ventilación puede verse afectada. En este nivel, los pacientes no pueden ser fácilmente excitados, pero responden a propósito después de una estimulación repetida o dolorosa. $\mathrm{La}$ anestesia general corresponde al último nivel, en el cual los pacientes no se pueden despertar, incluso por estimulación dolorosa. Este nivel se caracteriza principalmente por una ventilación espontánea deprimida, requiriendo ventilación artificial.

Los procedimientos quirúrgicos mínimamente invasivos, como es el caso de las endoscopias, requieren que los pacientes se encuentren entre los niveles 1 y 3 de sedación-analgesia (anestesia ligera), de manera que estos mantengan su función cardiovascular y ventilación espontánea, así como su capacidad de respuesta a estímulos verbales, táctiles o dolorosos. La monitorización continua de estos niveles de anestesia ligera es de gran importancia debido a que permite manejar de manera más adecuada la administración de fármacos, facilitando la labor del cirujano sin tener que llevar al paciente a niveles de anestesia general, al tiempo que reduce los niveles de estrés en los pacientes y mejora su proceso de recuperación.

En la actualidad existen varios sistemas de monitoreo que a partir de la señal del electroencefalograma (EEG) predicen el nivel de anestesia, entre ellos están el Bispectral analysis (BIS) [2], auditoryevoked potential (AEP) [3] y cerebral state index (CSI) [4]. Sin embargo, estos sistemas están diseñados para medir los niveles de anestesia general y algunos estudios $[5,6]$ indican que una de sus limitaciones es que no son capaces de distinguir de forma confiable entre niveles de anestesia ligera y anestesia profunda. Entre las razones, está la gran variabilidad de la señal EEG en el tiempo la cual es muy sensible al estado del paciente [7]. Además, la mayoría de estos sistemas se limitan al uso de técnicas de análisis espectral, lo cual requiere que la señal de entrada sea estacionaria. Esto provoca un retardo inevitable en la medición y hace que estos sistemas pierdan eficiencia cuando se trabaja con señales de dinámica compleja como lo son las señales cerebrales. Lo anterior, implica que estas técnicas no pueden detectar un cambio temprano en la actividad cerebral, afectando la detección oportuna del despertar perioperatorio.

Aunque estudios recientes podrían determinar cierta relación entre los cambios en la señal EEG, los niveles de anestesia ligera y la respuesta del paciente a estímulos dolorosos en el estado consciente e inconsciente inducido por los fármacos anestésicos [8], estos cambios en la señal EEG aún no han sido estudiados en profundidad. Es necesario ampliar el análisis de la señal EEG, usando métodos tanto en el dominio de la frecuencia como en el dominio del tiempo, con el fin de encontrar índices predictores robustos para determinar el nivel de anestesia ligera. Por otra parte, estos análisis también deberían incluir bandas de frecuencia superiores a las bandas tradicionales del EEG, las cuales han demostrado contener información útil al mejorar la discriminación entre diferentes niveles de anestesia ligera, utilizando técnicas basada en la densidad espectral de potencia y la función de información auto-mutua [9].

El gráfico de Poincaré es un método no lineal que permite analizar la variabilidad de una señal, dibujando dicha señal contra ella misma, pero con un tiempo de retardo. Algunos trabajos [10-13] indican que el gráfico de Poincaré es un método de gran interés debido a su capacidad para exhibir características no lineales de señales en el dominio del tiempo, y puede ser utilizado con datos no filtrados y no estacionarios. Por lo tanto, puede ser un método útil para analizar los procesos dinámicos complejos de la señal cerebral durante la anestesia, el sueño y la conciencia. Recientemente, este método ha sido aplicado al análisis del EEG para la medición de niveles de anestesia general [11-14]. Sin embargo, 
su aplicación en la medición de niveles de anestesia ligera aún no se ha abordado con profundidad.

En este artículo se analiza el efecto del tiempo de retardo del gráfico de Poincaré sobre la generación de índices para caracterizar el nivel de anestesia ligera en 110 pacientes sometidos a endoscopia digestiva. Posteriormente, se realiza un análisis multivariable con los mejores índices derivados del análisis de Poincaré y los parámetros obtenidos del análisis espectral de señales que contienen información del EEG y EMG (electromiografía) para modelar el nivel de anestesia ligera, teniendo como referencia la escala de sedación Ramsay [15]. Resultados preliminares, utilizando el grafico de Poincaré con un tiempo de retardo fijo, fueron presentados en [16].

\section{METODOLOGÍA}

\section{Gráfico de Poincaré}

El teorema de Takens establece que el atractor de un sistema dinámico puede ser reconstruido como una representación en el espacio de estado para un tiempo de retardo especifico (lag) y una dimensión (m). A pesar de que el atractor de un sistema dado puede tener una alta dimensión, el análisis de los atractores reconstruidos en dos dimensiones se ha utilizado ampliamente para caracterizar señales biomédicas [17]. El grafico de Poincaré, es un caso particular del teorema de Takens, en donde se elige $\mathrm{m}=2$. Es decir, corresponde a una reconstrucción en dos dimensiones con un retardo específico.

Dada una señal en el tiempo $x(i), i=1, \ldots, N$, el grafico de Poincaré corresponde a graficar $x(i)$ contra $x(i+\mathrm{lag})$, donde el parámetro lag es el tiempo de retardo entre las coordenadas $x$ y $y$ del plano cartesiano (ver Figura 1). El punto medio de la nube de puntos corresponde al promedio $(M x)$ de los voltajes de la señal $x(i)$. Dado un punto $p(V \mathrm{a}, V \mathrm{~b})$ en la nube de puntos del gráfico de Poincaré, la dispersión es cuantificada a partir de los valores $L_{1}$ y $L_{2}$, los cuales corresponden a la distancia desde el punto $p(V \mathrm{a}, V \mathrm{~b})$ a la línea de identidad (Eje 1) y a la línea perpendicular (Eje 2), respectivamente (Ver Figura 2). La cuantificación de la geometría del gráfico de Poincaré se realiza por medio de los índices $S D 1$ y $S D 2$, que pueden obtenerse a partir de las siguientes expresiones matemáticas:

$$
\begin{aligned}
& S D 1=\sqrt{\frac{\sum L_{1}^{2}}{N}} \\
& S D 2=\sqrt{\frac{\sum L_{2}^{2}}{N}}
\end{aligned}
$$

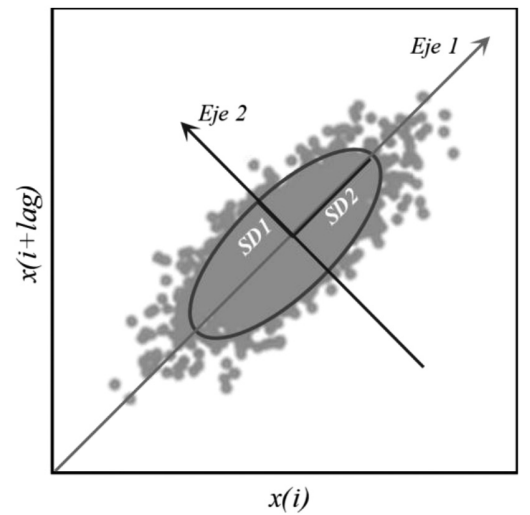

Figura 1. Gráfico de Poincaré.

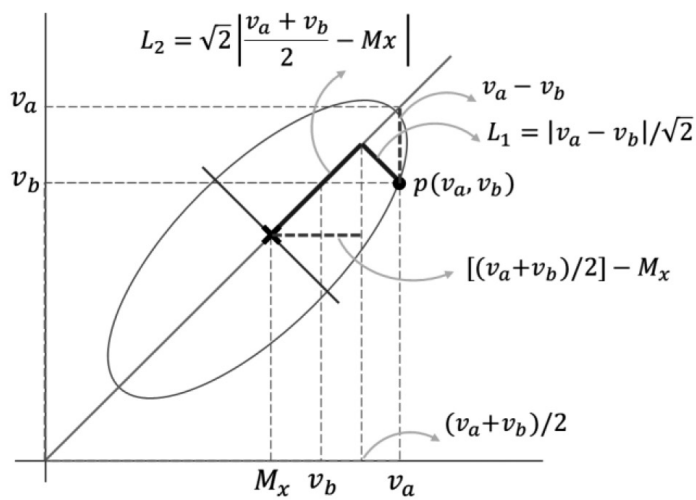

Figura 2. Expresiones aritméticas para cuantificar el gráfico de Poincaré.

En términos de estadística lineal, $S D 1$ mide la desviación estándar de los puntos perpendiculares a la línea de identidad y determina el ancho de la elipse (variabilidad a corto plazo), mientras que $S D 2$ mide la desviación estándar a lo largo de la línea de identidad y determina la longitud o elongación de la elipse (variabilidad a largo plazo) [14]. De igual forma, también es posible calcular la relación $S D 1 /$ $S D 2$ que representa la aleatoriedad de la señal, en otras palabras, esta relación caracteriza la forma alargada o asimetría del patrón de dispersión.

\section{Análisis del Tiempo de Retardo}

La forma del gráfico de Poincaré es función del tipo de señal y del tiempo de retardo utilizado en 
la construcción del gráfico. Si la señal a analizar presenta un cambio lento, es decir, que el próximo punto tiene aproximadamente el mismo valor que el anterior, el patrón de dispersión del gráfico de Poincaré presenta una forma más alargada. Por el contrario, si la señal presenta gran variabilidad, es decir que no hay una correlación entre el próximo punto y el anterior, el patrón de dispersión del gráfico de Poincaré presenta una forma circular. En la Figura 3 se observa el grafico de Poincaré de una señal aleatoria pura y de una señal aleatoria contaminada con una componente lineal $(y=0,1 x)$. Para estos casos, el tiempo de retardo no afecta la forma del patrón de dispersión, indicando que para el análisis de variabilidad de la señal teniendo en cuenta componentes lineales, el tiempo de retardo no tiene un efecto directo sobre los resultados. Sin embargo, cuando se analizan señales periódicas, la variación en el tiempo de retardo tiene un gran impacto. Si el tiempo de retardo es muy grande en comparación con el periodo de la señal, sus características oscilatorias no se verán reflejadas en el patrón de dispersión [14]. En la Figura 4 se presenta el gráfico de Poincaré de una señal sinusoidal de $10 \mathrm{~Hz}$ muestreada a 200 muestras por segundo, usando diferentes tiempos de retardo.
El patrón de dispersión obtenido a través del gráfico de Poincaré dependerá de la composición espectral de la señal a analizar. Por ejemplo, para una señal sinusoidal de frecuencia baja el tiempo de retardo en comparación con el periodo de la señal puede ser adecuado y por lo tanto generaría un patrón de dispersión alargado y caracterizado completamente por los índices $S D 1, S D 2$ y $S D 1 / S D 2$. Sin embargo, este mismo tiempo de retardo para una señal sinusoidal de alta frecuencia puede ser muy grande y el patrón de dispersión presentará una forma más circular, lo que erróneamente se podría interpretar como la existencia de una pobre correlación entre los valores de la señal sinusoidal. En la Figura 5 se muestra el gráfico de Poincaré de tres señales sinusoidales de diferentes frecuencias, distribuidas en un rango de 0-110 Hz, usando diferentes tiempos de retardo.

A partir de la Figura 5 es posible concluir que el tiempo de retardo óptimo para el gráfico de Poincaré de una señal, teniendo en cuenta su composición espectral, debe ser menor o igual a $1 / 5$ del periodo de la componente de mayor frecuencia de la señal (Para el ejemplo de la Figura 5, 1/5 equivale aproximadamente a 2 muestras). De esta manera se garantiza que todos sus componentes espectrales
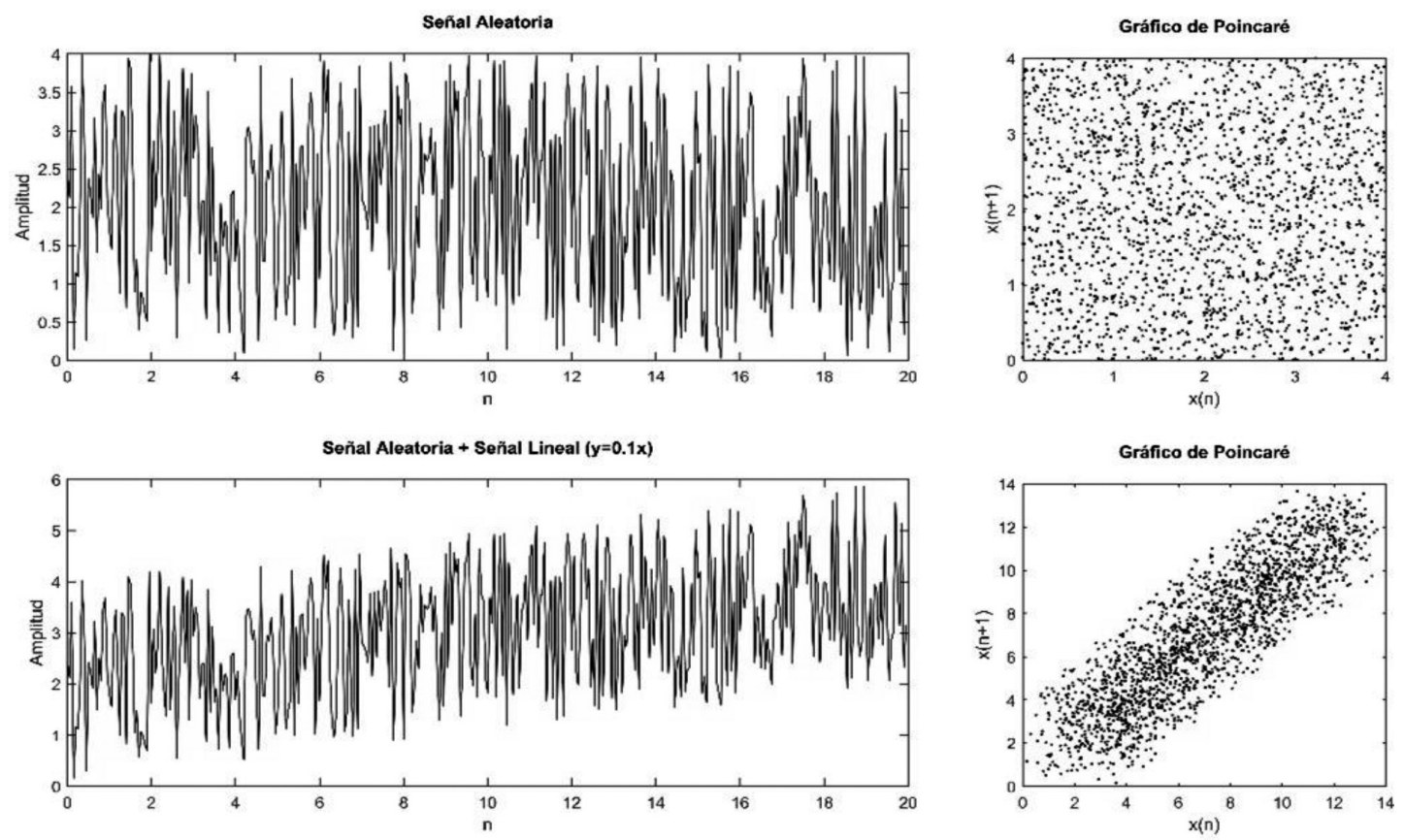

Figura 3. Gráfico de Poincaré de una señal aleatoria pura (panel superior) y de ella misma después de agregarle una componente lineal (panel inferior). 

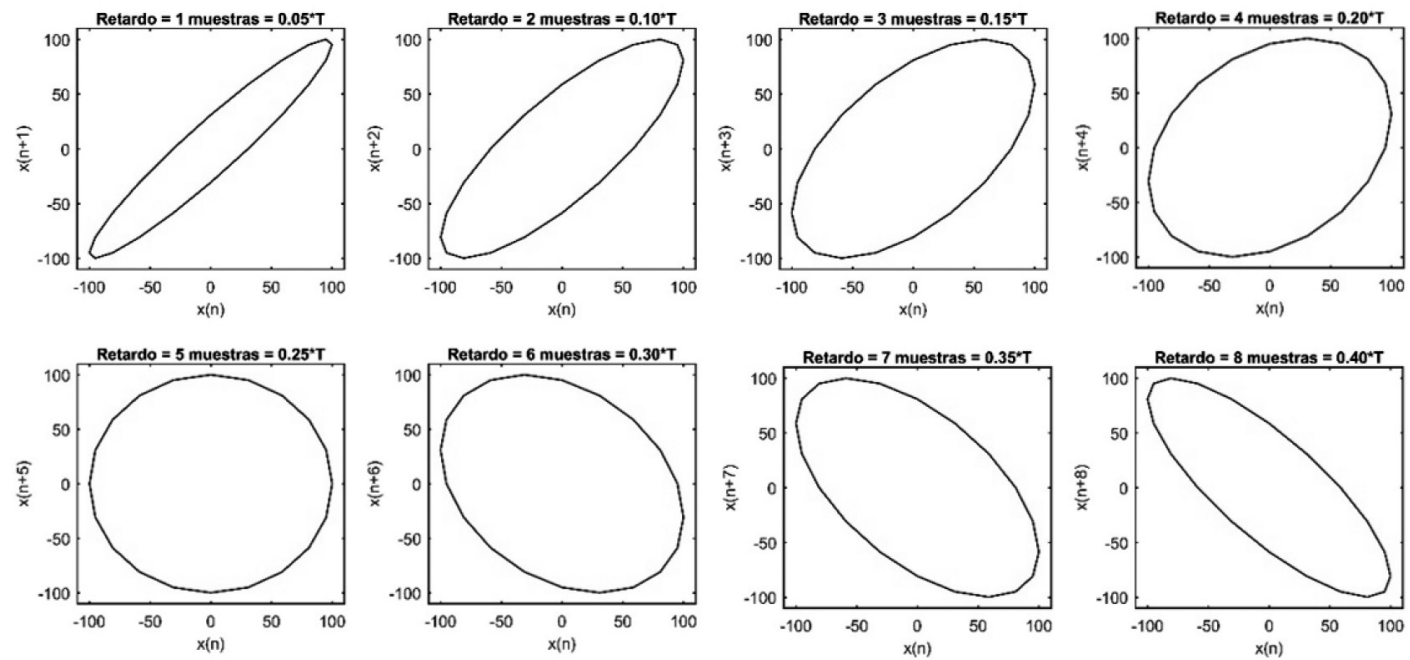

Figura 4. Efecto del retardo en el Gráfico de Poincaré de una señal sinusoidal de $10 \mathrm{~Hz}$ muestreada a 200 Hz. "T" es el periodo de la señal.
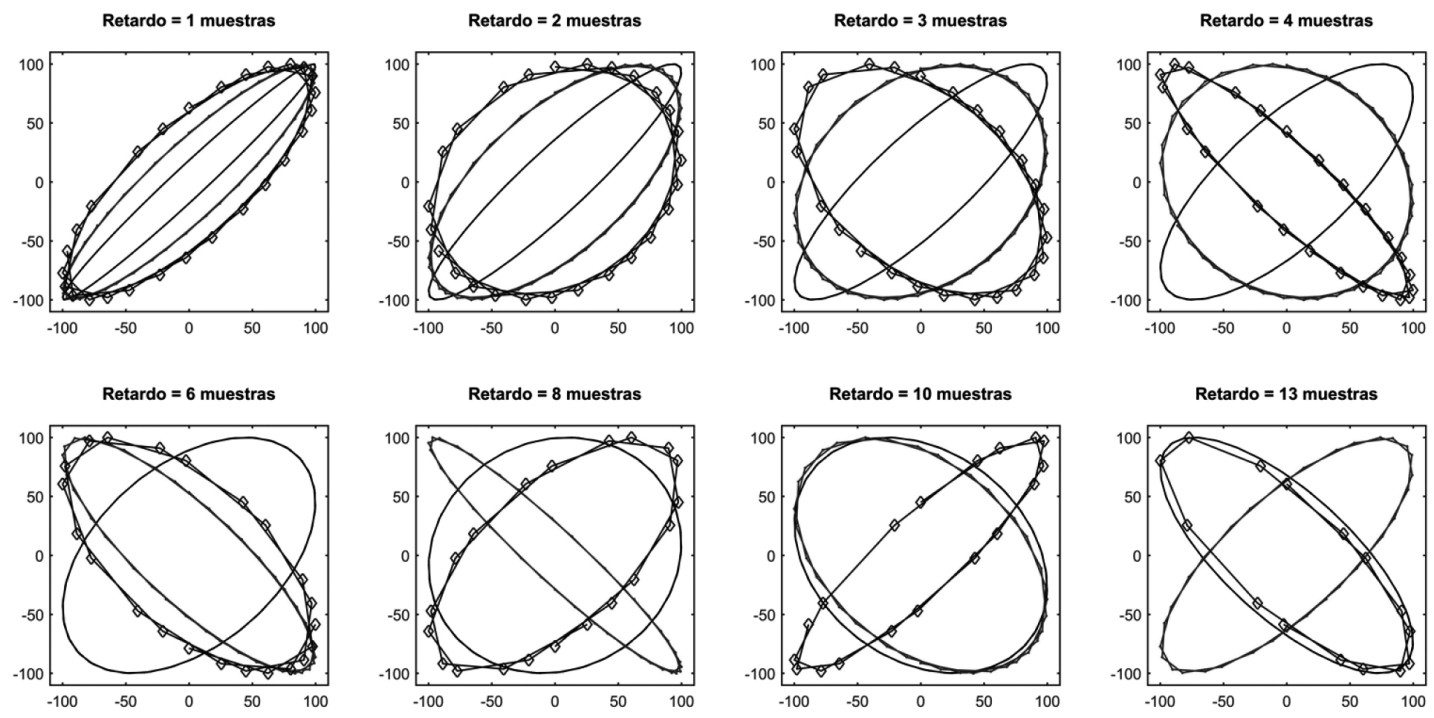

Figura 5. Efecto del retardo en el Gráfico de Poincaré para 3 señales sinusoidales con frecuencias entre $0-110 \mathrm{~Hz}(30 \mathrm{~Hz}, 70 \mathrm{~Hz}$ y $110 \mathrm{~Hz})$ muestreadas a $1024 \mathrm{~Hz}$.

presentarán un patrón de dispersión alargado que puede ser caracterizado por los índices $S D 1, S D 2$ y $S D 1 / S D 2$. Cabe mencionar que es posible obtener patrones similares del gráfico de Poincaré cuando el tiempo de retardo supera la duración del periodo de la señal, indicando una correlación (o anticorrelación) de las muestras de la señal en distintos periodos de tiempo. En el ejemplo mostrado en la Figura 5, en donde se han elegido rangos de frecuencias que serán objeto de estudio en este trabajo, este efecto se observa en la componente de $110 \mathrm{~Hz}$, la cual presenta un patrón similar cuando se utiliza un retardo de 1 muestra o de 10 muestras.

Finalmente, se realiza una prueba en donde se modifica la amplitud de una señal sinusoidal y se varía el retardo 
con el fin de observar los cambios en el patrón de dispersión en el gráfico de Poincaré. Los resultados obtenidos se muestran en la Figura 6. En este caso es posible observar que para un tiempo de retardo menor o igual a $1 / 5$ del periodo de la componente de mayor frecuencia de la señal, la variación en amplitud afecta en forma más significativa al índice $S D 2$ que al índice $S D 1$. Por otra parte, teniendo en cuenta los resultados de la Figura 5, es posible observar que el valor de $S D 1$ depende claramente de las variaciones de frecuencia de la señal. Por lo tanto, una onda de baja frecuencia con altas amplitudes generaría un valor bajo del índice $S D 1$ y un valor alto en el índice $S D 2$, esto es equivalente a un patrón de dispersión con una forma alargada.

\section{Bases de Datos}

La base de datos utilizada contiene información de 110 pacientes bajo anestesia ligera en procedimientos de endoscopia digestiva (ultrasonographic endoscopy -USE), y fue recolectada después de recibir la aprobación del comité de ética del hospital Clinic de Barcelona junto con un consentimiento firmado por los pacientes. La endoscopia digestiva es un procedimiento relativamente largo con periodos de estabilidad en el efecto de los fármacos, lo cual permite estudiar el efecto de estímulos dolorosos sobre el nivel de anestesia ligera. Cada paciente fue monitoreado en forma rutinaria, incluyendo el registro de las concentraciones en efecto de Propofol (CePropo) y de Remifentanil (CeRemi), las cuales fueron suministradas por un sistema de infusión TCI (Target Controlled Infusion - modelo Base Primea de Fresenius-Vial).

La adquisición de las señales que contienen información del EEG y EMG se realizó a través de un montaje de 3 electrodos (frente media (+), hueso malar (-), y frente izquierda (referencia)), con una frecuencia de muestreo de $900 \mathrm{~Hz}$, una resolución de 16 bits y una duración promedio de 60 minutos. Esta adquisición fue llevada a cabo por medio del monitor "AEP monitor/2" (Danmeter, Odense, Denmark). El índice BIS, obtenido a través de la señal EEG, fue monitoreado continuamente utilizando un monitor A2000 (versión de software 3,31 - Aspect Medical Systems, Newton, MA). Las respuestas categóricas observadas ante estímulos dolorosos fueron evaluadas usando la escala de sedación de Ramsay (Ramsay Sedation Score - RSS) [15]. Las características de la respuesta ante el estímulo nociceptivo en cada uno de los valores de la escala de Ramsay se observan en la Tabla 1. Los valores de RSS fueron estimados en tiempos aleatorios con el fin de evitar factores relacionados con el tiempo que pudieran afectar los resultados de las mediciones RSS. En este estudio, la base de datos contiene datos registrados de RSS con valores de 2 a 6 .

Tabla 1. Escala de Sedación de Ramsay.

\begin{tabular}{|c|l|}
\hline Valor & \multicolumn{1}{|c|}{ Descripción } \\
\hline 1 & Paciente despierto, ansioso, agitado o inquieto. \\
\hline 2 & $\begin{array}{l}\text { Paciente despierto, cooperativo, orientado y } \\
\text { tranquilo. }\end{array}$ \\
\hline 3 & $\begin{array}{l}\text { Paciente somnoliento con respuesta a comandos } \\
\text { verbales. }\end{array}$ \\
\hline 4 & $\begin{array}{l}\text { Paciente dormido, con rápida respuesta a la luz, } \\
\text { golpe ligero en la glabela o estímulo auditivo } \\
\text { intenso. }\end{array}$ \\
\hline 5 & $\begin{array}{l}\text { Paciente dormido, con respuesta lenta al } \\
\text { estimulo. }\end{array}$ \\
\hline 6 & $\begin{array}{l}\text { Sin respuesta a la presión fuerte sobre las } \\
\text { uñas o cualquier otro estimulo nociceptivo. }\end{array}$ \\
\hline
\end{tabular}
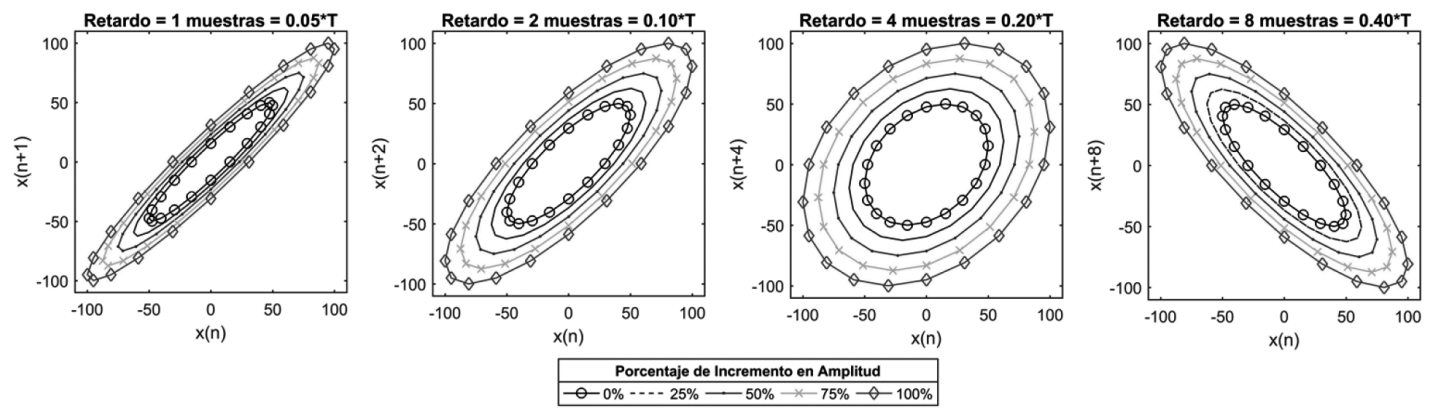

Figura 6. Efecto del retardo en el Gráfico de Poincaré de una señal sinusoidal de $10 \mathrm{~Hz}$ con diferentes amplitudes y muestreadas a $200 \mathrm{~Hz}$. "T" es el periodo de la señal. 


\section{Procesamiento de las Señales EEG}

Las señales que contienen información del EEG y EMG de la base de datos son re-muestreadas a una frecuencia de $1024 \mathrm{~Hz}$ para su análisis mediante el grafico de Poincaré. Todo dato de estos registros con un voltaje fuera del rango $+/-200 \mu \mathrm{V}$ se consideró como un artefacto [16] y fue eliminado.

Para llevar a cabo el análisis del nivel de anestesia ligera usando el gráfico de Poincaré, se utilizan ventanas de datos (Epochs) de 10 segundos, los cuales se superponen en un porcentaje igual al $90 \%$ entre Epochs consecutivos, permitiendo obtener datos de los índices SD1, SD2, y SD1/SD2 cada segundo. Este proceso se lleva acabo sobre tres rangos de frecuencia diferentes $(\mathrm{RF} 1=0,5-110 \mathrm{~Hz}, \mathrm{RF} 2=0,5-30 \mathrm{~Hz}$ y $\mathrm{RF} 3=30-110 \mathrm{~Hz}$ ) con el objetivo de estudiar el efecto tanto de las componentes propiamente dichas del EEG como de las componentes de la actividad muscular (EMG), la cual también está presente en los registros EEG. Mientras que el rango RF1 incluye tanto las componentes de baja frecuencia (EEG) como las de alta frecuencia (EMG), el rango FR2 permite hacer énfasis solo en los componentes más relacionados con la señal EEG y el rango FR3 con la señal de EMG [16].

Para el análisis del tiempo de retardo en el gráfico de Poincaré se utilizan seis valores de retardo, los cuales se aplican a cada rango de frecuencia. Los tiempos de retardo seleccionados son 1, 2, 4, 6, 8 y 10 muestras, que equivalen a $0,98 \mathrm{~ms}, 1,95 \mathrm{~ms}, 3,9 \mathrm{~ms}, 5,86 \mathrm{~ms}$, $7,81 \mathrm{~ms}$ y $9,77 \mathrm{~ms}$, respectivamente. Estos valores incluyen tiempos de retardo tanto inferiores como superiores al periodo de la componente de mayor frecuencia de este estudio $(110 \mathrm{~Hz})$. Lo anterior con el fin de validar la elección de un tiempo de retardo adecuado en la predicción del nivel de anestesia ligera.

Los mismos Epochs que se usan para el análisis de Poincaré, también son utilizados para un análisis espectral de potencia. Para este análisis, los datos son sub-muestreados a una frecuencia de $256 \mathrm{~Hz}$, previa aplicación de un filtro antialiasing paso bajo Chebyshev de sexto orden con frecuencia de corte de $128 \mathrm{~Hz}$. De esta manera se garantizan señales con bandas de frecuencia superiores a las bandas tradicionales del EEG, y se reduce el número de muestras por segundo para mejorar la eficiencia de cómputo del algoritmo de la transformada rápida de Fourier [18]. La densidad espectral de potencia (Power Spectral Density - PSD) para cada Epoch se obtiene después de aplicar una ventana de Hamming sobre los datos. La potencia espectral por bandas de frecuencia es calculada como el área bajo la curva normalizada de la PSD para cada uno de los rangos de frecuencia dados [16]: delta ( $\mathrm{P} \delta, 0,1-4 \mathrm{~Hz})$, teta $(\mathrm{P} \theta$, 4-8 Hz), alfa $(\mathrm{P} \alpha, 8-12 \mathrm{~Hz})$ y beta $(\mathrm{P} \beta, 12-30 \mathrm{~Hz})$.

Dentro del análisis espectral también se calculan la entropía espectral de la señal ( $\mathrm{SpEn}$ ) y la frecuencia límite a 95\% (SEF_95). La entropía espectral cuantifica el valor esperado de la información contenida en una señal en el espectro de frecuencias. El valor de la entropía espectral será bajo cuando la potencia de la señal se concentre en un pequeño conjunto de frecuencias, e incrementará de acuerdo a la uniformidad en que los valores de amplitud se dispersen en los componentes de frecuencia de la señal. Por lo tanto, la entropía espectral puede ser vista como una medida de la dispersión de la potencia de la señal en el espectro de frecuencias. La frecuencia límite de $95 \%$ (SEF_95\%) representa la frecuencia por debajo de la cual se encuentra el 95\% de la potencia total del espectro de frecuencias. A medida que incrementa el grado de anestesia, el valor del índice SEF_95\% decrementa debido a que la potencia de la señal EEG se desplaza hacia las frecuencias bajas [19].

\section{Análisis Estadístico}

Los parámetros obtenidos del EEG (índices derivados del mapa de Poincaré y del análisis espectral), así como las variables CePropo, CeRemi y BIS, fueron asociados a los correspondientes valores observados de la escala Ramsay (RSS). Considerando que el nivel de sedación en un paciente debería de permanecer sin cambios si las concentraciones de fármacos permanecen constantes, para el análisis estadístico se consideraron ventanas de 1 minuto de duración tomadas justo antes del instante en que se anotó la respuesta RSS, siempre y cuando CePropo y CeRemi fueran constantes en dicha ventana. En este trabajo, CePropo y CeRemi fueron consideradas constantes si la variación de ellas (DCePropo y DCeRemi), entre el primer y último segundo de la ventana de 1 minuto, fue: $\Delta$ CeRemi $<0,1 \mathrm{ng} / \mathrm{mL}$ y $\Delta$ CeProp $<0,1 \mu \mathrm{g} /$ $\mathrm{mL}$. En caso contrario, la ventana se recortó hasta la muestra en la cual se cumplían las condiciones.

En este estudio se llevaron a cabo dos procesos de análisis. En el Análisis 1, se tienen en cuenta los datos relacionados con todos los niveles de la escala de Ramsay (RSS2, RSS3, RSS4, RSS5 y RSS6). 
En el Análisis 2, solo se consideraron los datos relacionados con los niveles RSS5 y RSS6, que corresponden a la respuesta categórica observada después de un estímulo nociceptivo [16].

Para determinar la capacidad de predicción del nivel de anestesia ligera teniendo en cuenta la escala de sedación de Ramsay, se realiza un análisis comparativo de los índices calculados utilizando el estadístico de probabilidad de predicción (Pk) [20]. El valor Pk es una herramienta estadística utilizada para medir que tan bien un índice predice una variable de salida, que en este caso es el estado del paciente dado por la escala Ramsay. Un valor de Pk igual a 1 indica una predicción perfecta, mientras que un valor de 0,5 indica una capacidad predictiva nula.

Adicionalmente, se utilizó un sistema de inferencia neuro-difuso adaptativo (Adaptive Neuro-Fuzzy Inference System-ANFIS) para modelar la interacción de los mejores índices con respecto al valor del RSS en cada uno de los procesos de análisis. El proceso de validación se realiza usando el método de validación cruzada (dejando uno fuera). Para el Análisis 2 también se obtuvo el estadístico de exactitud (Acc-accuracy). Para el procesamiento de las señales y posterior análisis estadístico se utilizó el software MATLAB $^{\text {TM }}$ (MathWorks, Inc., Natick, MA).

\section{RESULTADOS}

En esta sección se presentan los resultados del Análisis 1 y 2, correspondientes a la evaluación de la capacidad predictiva (Pk) del nivel de anestesia ligera de cada uno de los índices calculados, respectivamente. Con el fin de reducir la cantidad de datos, solo se muestran aquellos índices que obtuvieron un $\mathrm{Pk}>0,6$, a excepción del SEF95 y SpEn que se incluyen a modo de referencia. Para cada índice se indica su valor promedio y la desviación estándar en cada una de las escalas de anestesia ligera.

\section{Medición del nivel de Sedación}

A continuación, se presentan los resultados del Análisis 1. En la Tabla 2 se encuentran los valores de las concentraciones de fármacos y de los índices derivados del análisis espectral, además del valor del BIS. Los resultados de los índices obtenidos a partir del gráfico de Poincaré para los rangos de frecuencia RF1, RF2 y RF3 se presentan en las Tablas 3, 4 y 5, respectivamente, considerando diferentes tiempos de retardo.

De la Tabla 2 se observa que los mejores valores de Pk fueron obtenidos con el BIS $(0,775), \mathrm{P} \alpha(0,757)$ y CePropo $(0,719)$, mientras que SEF95 y SpEn

Tabla 2. Capacidad Predictiva del Nivel de Sedación (Pk): Análisis Espectral y Concentraciones de Fármacos.

\begin{tabular}{|l|c|c|c|c|c|c|}
\hline \multicolumn{1}{|c|}{ Índice } & $\begin{array}{c}\text { RSS2 } \\
\text { mean } \pm \text { std }\end{array}$ & $\begin{array}{c}\text { RSS3 } \\
\text { mean } \pm \text { std }\end{array}$ & $\begin{array}{c}\text { RSS4 } \\
\text { mean } \pm \text { std }\end{array}$ & $\begin{array}{c}\text { RSS5 } \\
\text { mean } \pm \text { std }\end{array}$ & $\begin{array}{c}\text { RSS6 } \\
\text { mean } \pm \text { std }\end{array}$ & Pk \\
\hline BIS & $93,0 \pm 7,65$ & $82,1 \pm 10,8$ & $74 \pm 12,1$ & $65,6 \pm 14,5$ & $64,9 \pm 14,8$ & 0,775 \\
\hline CeRemi & $0,566 \pm 0,888$ & $1,11 \pm 0,824$ & $1,25 \pm 0,817$ & $0,984 \pm 0,899$ & $1,47 \pm 0,72$ & 0,615 \\
\hline CePropo & $0,525 \pm 0,79$ & $1,88 \pm 0,715$ & $2,15 \pm 0,676$ & $2,38 \pm 0,652$ & $2,21 \pm 0,632$ & 0,719 \\
\hline Pd & $0,539 \pm 0,224$ & $0,296 \pm 0,210$ & $0,242 \pm 0,165$ & $0,23 \pm 0,168$ & $0,277 \pm 0,169$ & 0,629 \\
\hline Pa & $0,085 \pm 0,071$ & $0,178 \pm 0,109$ & $0,260 \pm 0,135$ & $0,318 \pm 0,142$ & $0,322 \pm 0,146$ & 0,757 \\
\hline SEF95 & $18,2 \pm 7,27$ & $22,4 \pm 4,43$ & $20,6 \pm 3,70$ & $18,9 \pm 3,10$ & $18,3 \pm 2,98$ & 0,585 \\
\hline SpEn & $0,777 \pm 0,081$ & $0,845 \pm 0,062$ & $0,84 \pm 0,049$ & $0,826 \pm 0,047$ & $0,818 \pm 0,041$ & 0,502 \\
\hline
\end{tabular}

Tabla 3. Capacidad Predictiva del Nivel de Sedación (Pk): Gráfico de Poincaré en el rango RF1 = 0,5-110 Hz.

\begin{tabular}{|l|c|c|c|c|c|c|c|}
\hline \multicolumn{1}{|c|}{ Índice } & $\begin{array}{c}\text { Retardo } \\
\text { muestras }\end{array}$ & $\begin{array}{c}\text { RSS2 } \\
\text { mean } \pm \text { std }\end{array}$ & $\begin{array}{c}\text { RSS3 } \\
\text { mean } \pm \text { std }\end{array}$ & $\begin{array}{c}\text { RSS4 } \\
\text { mean } \pm \text { std }\end{array}$ & $\begin{array}{c}\text { RSS5 } \\
\text { mean } \pm \text { std }\end{array}$ & $\begin{array}{c}\text { RSS6 } \\
\text { mean } \pm \text { std }\end{array}$ & Pk \\
\hline SD1RF1 & 10 & $8,90 \pm 5,90$ & $6,91 \pm 4,77$ & $5,81 \pm 3,67$ & $5,44 \pm 2,64$ & $4,14 \pm 2,36$ & 0,667 \\
\hline \multirow{2}{*}{ SD2RF1 } & 4 & $22,0 \pm 11,7$ & $14,4 \pm 6,53$ & $14,3 \pm 6,48$ & $15,1 \pm 5,71$ & $12,2 \pm 5,74$ & 0,620 \\
\cline { 2 - 8 } & 6 & $21,0 \pm 11,6$ & $13,5 \pm 6,08$ & $13,6 \pm 6,23$ & $14,5 \pm 5,56$ & $11,6 \pm 5,55$ & 0,615 \\
\hline SD1/SD2RF1 & 10 & $0,422 \pm 0,220$ & $0,426 \pm 0,196$ & $0,336 \pm 0,158$ & $0,299 \pm 0,103$ & $0,270 \pm 0,109$ & 0,631 \\
\hline
\end{tabular}


Tabla 4. Capacidad Predictiva del Nivel de Sedación (Pk): Gráfico de Poincaré en el rango RF2 = 0,5-30 Hz.

\begin{tabular}{|c|c|c|c|c|c|c|c|}
\hline Índice & $\begin{array}{c}\text { Retardo } \\
\text { muestras }\end{array}$ & $\begin{array}{c}\text { RSS2 } \\
\text { mean } \pm \text { std }\end{array}$ & $\begin{array}{c}\text { RSS3 } \\
\text { mean } \pm \text { std }\end{array}$ & $\begin{array}{c}\text { RSS4 } \\
\text { mean } \pm \text { std }\end{array}$ & $\begin{array}{c}\text { RSS5 } \\
\text { mean } \pm \text { std }\end{array}$ & $\begin{array}{c}\text { RSS6 } \\
\text { mean } \pm \text { std }\end{array}$ & Pk \\
\hline \multirow{4}{*}{ SD2RF2 } & 1 & $20,0 \pm 11,6$ & $12,5 \pm 5,88$ & $12,9 \pm 6,20$ & $13,9 \pm 5,64$ & $10,9 \pm 5,52$ & 0,606 \\
\cline { 2 - 8 } & 2 & $19,9 \pm 11,6$ & $12,5 \pm 5,87$ & $12,8 \pm 6,19$ & $13,9 \pm 5,63$ & $10,9 \pm 5,51$ & 0,606 \\
\cline { 2 - 8 } & 4 & $19,9 \pm 11,7$ & $12,4 \pm 5,86$ & $12,8 \pm 6,17$ & $13,8 \pm 5,61$ & $10,9 \pm 5,49$ & 0,606 \\
\cline { 2 - 8 } & 6 & $19,8 \pm 11,7$ & $12,3 \pm 5,84$ & $12,6 \pm 6,14$ & $13,7 \pm 5,57$ & $10,8 \pm 5,44$ & 0,605 \\
\cline { 2 - 8 } & 10 & $19,6 \pm 11,7$ & $12,1 \pm 5,81$ & $12,4 \pm 6,10$ & $13,5 \pm 5,51$ & $10,6 \pm 5,38$ & 0,605 \\
\hline
\end{tabular}

Tabla 5. Capacidad Predictiva del Nivel de Sedación (Pk): Gráfico de Poincaré en el rango RF3 = 30-110 Hz.

\begin{tabular}{|l|c|c|c|c|c|c|c|}
\hline \multicolumn{1}{|c}{ Índice } & $\begin{array}{c}\text { Retardo } \\
\text { muestras }\end{array}$ & $\begin{array}{c}\text { RSS2 } \\
\text { mean } \pm \text { std }\end{array}$ & $\begin{array}{c}\mathbf{R S S 3} \\
\text { mean } \pm \text { std }\end{array}$ & $\begin{array}{c}\mathbf{R S S 4} \\
\text { mean } \pm \text { std }\end{array}$ & $\begin{array}{c}\text { RSS5 } \\
\text { mean } \pm \text { std }\end{array}$ & $\begin{array}{c}\text { RSS6 } \\
\text { mean } \pm \text { std }\end{array}$ & Pk \\
\hline SD1RF3 & 10 & $7,44 \pm 5,84$ & $4,96 \pm 4,84$ & $3,46 \pm 3,70$ & $2,72 \pm 2,55$ & $2,31 \pm 1,93$ & 0,705 \\
\hline \multirow{5}{*}{ SD1/SD2 RF3 } & 1 & $0,250 \pm 0,032$ & $0,270 \pm 0,038$ & $0,289 \pm 0,033$ & $0,289 \pm 0,04$ & $0,296 \pm 0,031$ & 0,684 \\
\cline { 2 - 8 } & 2 & $0,530 \pm 0,08$ & $0,582 \pm 0,095$ & $0,630 \pm 0,085$ & $0,632 \pm 0,101$ & $0,650 \pm 0,079$ & 0,685 \\
\cline { 2 - 8 } & 4 & $1,42 \pm 0,445$ & $1,74 \pm 0,607$ & $2,09 \pm 0,610$ & $2,16 \pm 0,650$ & $2,26 \pm 0,569$ & 0,686 \\
\cline { 2 - 8 } & 6 & $2,21 \pm 0,480$ & $2,49 \pm 0,664$ & $2,84 \pm 0,678$ & $2,90 \pm 0,761$ & $3,02 \pm 0,630$ & 0,670 \\
\cline { 2 - 8 } & 8 & $1,41 \pm 0,323$ & $1,17 \pm 0,317$ & $1,01 \pm 0,258$ & $0,974 \pm 0,228$ & $0,963 \pm 0,245$ & 0,711 \\
\cline { 2 - 8 } & 10 & $0,884 \pm 0,347$ & $0,643 \pm 0,394$ & $0,434 \pm 0,353$ & $0,409 \pm 0,392$ & $0,353 \pm 0,343$ & 0,695 \\
\hline
\end{tabular}

mostraron valores de $\mathrm{Pk}<0,6$. Por otra parte, en las Tablas 3, 4 y 5 se observa que los mejores valores de predicción Pk se obtienen cuando se analiza el rango de altas frecuencias de la señal (Tabla 5, RF3 = 30-110 Hz). Particularmente, el índice SD1/ $\mathrm{SD} 2 \mathrm{RF} 3$ presentó valores de $\mathrm{Pk}>0,65$ para todos los retardos estudiados, siendo el mejor Pk $(0,711)$ el obtenido con un retardo de 8 muestras. Para este mismo rango de altas frecuencias, el índice SD1RF3 también obtuvo su mejor valor de $\mathrm{Pk}=0,705$ utilizando un retardo de 10 muestras. Para el análisis que considera todas las componentes de frecuencia de la señal registrada (Tabla 3, RF1 $=0,5-110 \mathrm{~Hz}$ ), a excepción del índice SD1RF1 que obtuvo un
$\mathrm{Pk}=0,667$ (retardo de 10 muestras), los demás índices tuvieron valores de $\mathrm{Pk}<0,632$. Para el rango de bajas frecuencias (Tabla 4, RF2 $=0,5-30 \mathrm{~Hz}$ ) los índices presentaron los valores de Pk más bajos $(\mathrm{Pk}<0,61) \sin$ importar el valor del retardo.

Los índices que presentaron mayor capacidad predictiva, es decir, CePropo, P $\alpha$, SD1RF3 (retardo $=10$ muestras) y SD1/SD2RF3 (retardo $=8$ muestras), se utilizan como entradas de un sistema ANFIS para modelar los valores de RSS. Con estas entradas, el sistema ANFIS arroja una salida con la que se obtiene un valor $\mathrm{Pk}=0,799$. En la Figura 7 se observa la respuesta del sistema ANFIS a lo largo

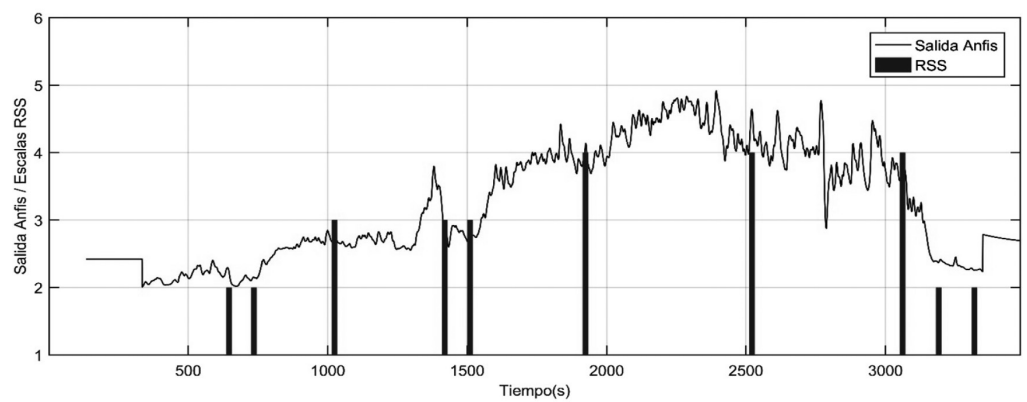

Figura 7. Respuesta del modelo ANFIS para determinar el nivel de anestesia ligera en función de las respuestas categóricas (escala RSS). 
del tiempo, junto con las respuestas categóricas observadas ante estímulos dolorosos de uno de los pacientes de la base de datos.

\section{Medición del nivel de Analgesia}

A continuación, se presentan los resultados para el Análisis 2, el cual solo compara las escalas RSS5 y RSS6 que están más directamente relacionadas con la respuesta a un estímulo dolorosos. En la Tabla 6 se presentan los resultados de los índices obtenidos a partir del análisis espectral y de las concentraciones de fármacos, así como el valor del BIS. Los resultados de los índices obtenidos a partir del gráfico de Poincaré para los rangos de frecuencia RF1 y RF2 se presentan en las Tablas 7 y 8 , respectivamente, considerando diferentes tiempos de retardo. Para el rango de alta frecuencia $(\mathrm{RF} 3=30-110 \mathrm{~Hz}$ ), ninguno de los índices derivados del gráfico de Poincaré presentó valores de Pk mayores a 0,6.

De la Tabla 6 se observa que, a diferencia de lo observado en el Análisis 1 (Tabla 2) donde el BIS obtuvo el mejor valor de Pk, para el Análisis 2 el BIS obtuvo un valor de $\mathrm{Pk}$ muy bajo $(\mathrm{Pk}=0,518)$, indicando que este índice prácticamente no tuvo ninguna capacidad de predicción de la respuesta de los pacientes ante los estímulos nociceptivos. El mejor valor de $\mathrm{Pk}$ se obtuvo con el índice CeRemi $(0,675)$, y nuevamente SEF95 y SpEn mostraron valores de $\mathrm{Pk}<0,6$. El parámetro espectral con mejor capacidad de predicción fue el índice $\mathrm{P} \beta$ $(\mathrm{Pk}=0,643)$. El índice Pa, el cual tuvo uno de los mejores valores de Pk en el Análisis 1 (Tabla 2), no tuvo ninguna capacidad de predicción de la respuesta de los pacientes ante los estímulos nociceptivos.

De la Tabla $7(\mathrm{RF} 1=0,5-110 \mathrm{~Hz})$ y Tabla 8 $(\mathrm{RF} 2=0,5-30 \mathrm{~Hz})$ se observa que los mejores valores de Pk se obtuvieron con los índices SD1 y SD2, siendo los valores de la Tabla 8 ligeramente mayores. Aunque los valores de $\mathrm{Pk}$ son muy similares para los diferentes tiempos de retardo, de la Tabla 8 se seleccionan los índices SD1RF2 (Retardo $=6$ muestras) y SD2RF2 (Retardo $=2$ muestras) como los mejores.

Los índices que presentan mayor valor $\mathrm{Pk}$, en este caso, CeRemi, P $\beta, \mathrm{SD} 1 \mathrm{RF} 2$ (Retardo $=6$ muestras) y SD2RF2 (Retardo = 2 muestras), se utilizan como entradas de un sistema ANFIS con el fin de predecir los valores RSS5 y RSS6 de la escala de Ramsay. Con estas entradas, el sistema ANFIS genera una

Tabla 6. Capacidad Predictiva del Nivel de Analgesia (Pk): Análisis Espectral y Concentraciones de Fármacos.

\begin{tabular}{|l|c|c|c|}
\hline Indice & RSS5 mean \pm std & RSS6 mean \pm std & Pk \\
\hline BIS & $65,6 \pm 14,5$ & $64,9 \pm 14,8$ & 0,518 \\
\hline CeRemi & $0,984 \pm 0,899$ & $1,47 \pm 0,72$ & 0,675 \\
\hline P & $0,23 \pm 0,168$ & $0,277 \pm 0,169$ & 0,600 \\
\hline P $\beta$ & $0,288 \pm 0,154$ & $0,218 \pm 0,130$ & 0,643 \\
\hline SEF95 & $18,9 \pm 3,10$ & $18,3 \pm 2,98$ & 0,549 \\
\hline SpEn & $0,826 \pm 0,047$ & $0,818 \pm 0,041$ & 0,575 \\
\hline
\end{tabular}

Tabla 7. Capacidad Predictiva del Nivel de Analgesia (Pk): Gráfico de Poincaré en el rango $\mathrm{RF} 1=0,5-110 \mathrm{~Hz}$.

\begin{tabular}{|c|c|c|c|c|}
\hline Indice & Retardo muestras & RSS5 mean \pm std & RSS6 mean \pm std & Pk \\
\hline SD1RF1 & 10 & $5,44 \pm 2,64$ & $4,14 \pm 2,36$ & 0,692 \\
\hline \multirow{4}{*}{ SD2RF1 } & 1 & $19,7 \pm 8,04$ & $17,1 \pm 8,88$ & 0,621 \\
\cline { 2 - 5 } & 2 & $18,6 \pm 7,23$ & $15,9 \pm 7,90$ & 0,633 \\
\cline { 2 - 5 } & 4 & $15,1 \pm 5,71$ & $12,2 \pm 5,74$ & 0,686 \\
\cline { 2 - 5 } & 6 & $14,5 \pm 5,56$ & $11,6 \pm 5,55$ & 0,690 \\
\cline { 2 - 5 } & 10 & $17,6 \pm 6,75$ & $15,1 \pm 7,46$ & 0,634 \\
\hline
\end{tabular}


Tabla 8. Capacidad Predictiva del Nivel de Analgesia (Pk): Gráfico de Poincaré en el rango $\mathrm{RF} 2=0,5-30 \mathrm{~Hz}$.

\begin{tabular}{|c|c|c|c|c|}
\hline Indice & Retardo muestras & RSS5 mean \pm std & RSS6 mean \pm std & Pk \\
\hline \multirow{4}{*}{ SD1RF2 } & 1 & $0,474 \pm 0,175$ & $0,36 \pm 0,174$ & 0,695 \\
\cline { 2 - 5 } & 2 & $0,941 \pm 0,347$ & $0,713 \pm 0,347$ & 0,703 \\
\cline { 2 - 5 } & 4 & $1,84 \pm 0,684$ & $1,38 \pm 0,681$ & 0,708 \\
\cline { 2 - 5 } & 6 & $2,68 \pm 1,02$ & $1,99 \pm 1,00$ & 0,711 \\
\cline { 2 - 5 } & 8 & $3,49 \pm 1,35$ & $2,57 \pm 1,32$ & 0,710 \\
\hline \multirow{4}{*}{ SD2RF2 } & 10 & $4,28 \pm 1,67$ & $3,15 \pm 1,64$ & 0,710 \\
\cline { 2 - 5 } & 1 & $13,9 \pm 5,64$ & $10,9 \pm 5,52$ & 0,697 \\
\cline { 2 - 5 } & 2 & $13,9 \pm 5,63$ & $10,9 \pm 5,51$ & 0,698 \\
\cline { 2 - 5 } & 4 & $13,8 \pm 5,61$ & $10,9 \pm 5,49$ & 0,697 \\
\cline { 2 - 5 } & 6 & $13,7 \pm 5,57$ & $10,8 \pm 5,44$ & 0,697 \\
\hline \multirow{3}{*}{ SD1/SD2 RF2 } & 10 & $13,5 \pm 5,51$ & $10,6 \pm 5,38$ & 0,697 \\
\cline { 2 - 5 } & 6 & $0,198 \pm 0,034$ & $0,186 \pm 0,034$ & 0,611 \\
\cline { 2 - 5 } & 8 & $0,261 \pm 0,047$ & $0,242 \pm 0,044$ & 0,624 \\
\hline & 10 & $0,326 \pm 0,061$ & $0,301 \pm 0,056$ & 0,632 \\
\hline
\end{tabular}

salida con la que se obtiene un $\mathrm{Pk}=0,78$ y un $\mathrm{Acc}=70,9 \%$. Esto permite modelar la respuesta de un paciente a estímulos nociceptivos, determinando la presencia o ausencia de dolor en el paciente.

\section{DISCUSIÓN}

Estudios recientes [11, 14 y 16] han demostrado que a través del análisis de la señal EEG por medio del gráfico de Poincaré es posible detectar los cambios de estado a medida que incrementa el grado de anestesia de un paciente, ya que la variabilidad a corto plazo de la señal EEG y la aleatoriedad se ven reducidas al incrementar el nivel de anestesia. Lo anterior afecta los valores de los índices SD1, $\mathrm{SD} 2$ y SD1/SD2, derivados del gráfico de Poincaré de la señal EEG.

Los resultados de la medición del nivel de anestesia ligera de acuerdo con las escalas de sedación de Ramsay, muestran que el índice SD1/SD2RF3, calculado a partir de la señal registrada en la frente de los pacientes (EEG+EMG) en un rango de frecuencia de $30-110 \mathrm{~Hz}$, ofrece información útil relacionada con el nivel de anestesia ligera en un paciente. En general, se observó que el índice SD1/SD2FR3 presentó una disminución significativa a medida que el valor de la escala Ramsay se incrementa desde RSS2 hasta RSS6. El retardo con el que el índice SD1/SD2RF3 alcanza la mayor capacidad predictiva
Pk es de 8 muestras (Tabla 5). Una posible razón que explica por qué el rango de alta frecuencia (RF3) es el que más información suministra en relación con la estimación del nivel de anestesia ligera, es el hecho de que los pacientes considerados en este estudio están sometidos a anestesia ligera y no a anestesia general. Por tal motivo, los pacientes que pertenecen a los grupos con $\mathrm{RSS}<=5$ presentan una mayor actividad muscular de la cara y del cuero cabelludo, la cual se ve fuertemente reflejada en la señal registrada. Estos resultados concuerdan con lo publicado en [21], donde se indicó que la electromiografía de la superficie facial es un buen indicador para discriminar entre una anestesia adecuada o inadecuada, siendo también útil en la medición del efecto de los fármacos, de los niveles de vigilancia y de la integridad del sistema nervioso central. Por su parte, Hayashi [11] presenta un análisis del gráfico de Poincaré en pacientes sometidos a anestesia general, donde se indica que el índice SD1/SD2, calculado en un rango de frecuencia de 0-30 Hz, podría no estar necesariamente relacionado con el nivel de anestesia en pacientes ligeramente anestesiados, y por el contrario, el índice SD2 podría estimar de forma más precisa estos niveles de anestesia poco profunda (anestesia ligera). En el presente trabajo, tal como sugiere Hayashi, el índice SD1/SD2RF2 no fue capaz de estimar el nivel de anestesia ligera $(\mathrm{Pk}=0,52-0,55)$, pero tampoco lo hizo el índice SD2RF2 (Pk = 0,604-0,606) 
(Tabla 4). Sin embargo, el índice SD1/SD2RF3 con un retardo de 8 muestras obtuvo un coeficiente $\mathrm{Pk}=0,711$, surgiendo como uno de los índices a ser considerado en la medición del nivel de anestesia ligera. Lo anterior sugiere que aunque el índice SD1/SD2 obtenido entre $0-30 \mathrm{~Hz}$ por Hayashi puede ser utilizado como indicador del nivel de anestesia general, este pierde capacidad predictiva $(\mathrm{Pk}=0,52-0,55)$ cuando se utiliza para medir el nivel de anestesia ligera. Sin embargo, este mismo índice SD1/SD2 mejora su capacidad de predicción del nivel de anestesia ligera $(\mathrm{Pk}=0,711)$ si se calcula sobre la banda de $30-110 \mathrm{~Hz}$, donde existe mayor componente de actividad EMG.

Los índices SD1RF2 con un retardo de 6 muestras y SD2RF2 con un retardo de 2 muestras obtuvieron el mejor rendimiento en la clasificación de las respuestas categóricas en los grupos RSS5 y RSS6 con un valor $\mathrm{Pk}=0,711$ y $\mathrm{Pk}=0,698$, respectivamente. Es importante mencionar que SD1RF2 incluye información que está relacionada con las variaciones de alta frecuencia (actividad en las bandas $\alpha$ y $\beta$ ) de la actividad del cerebro registrada en el rango de 0,5-30 Hz (RF2). Por otra parte, SD2RF2 depende en especial de las variaciones en amplitud de la señal EEG, lo cual ocurre principalmente cuando la actividad del EEG se mueve de la banda $\alpha$ hacia la banda $\theta$ [14]. También se debe señalar que en la presente base de datos, la señal EEG no contiene una actividad fuerte en la banda $\delta$, debido a que la banda $\delta$ está presente principalmente en casos de anestesia general, mientras que los pacientes del presente estudio solo llegan a niveles donde el BIS promedio es de 64,9 en la escala RSS6, la cual representa el estado de anestesia ligera más profundo sin llegar a ser un nivel de anestesia general.

Los índices espectrales como SEF95 y SpEn no presentaron valores de $\mathrm{Pk}$ altos, ni en el Análisis 1 ( 0,59 y 0,50 , respectivamente), ni en el Análisis 2 ( 0,54 y 0,57 , respectivamente). Lo cual sugiere que estos índices espectrales no tienen un buen desempeño cuando son utilizados en pacientes con anestesia ligera. Por otra parte, los índices BIS y CePropo presentaron un comportamiento similar, siendo un buen estimador del nivel de las escalas RSS para el Análisis 1 pero no para el Análisis 2, mientras que el índice CeRemi fue un mejor estimador en el Análisis 2 que en el Análisis 1. Esto era de esperarse ya que el Remifentanil es el fármaco analgésico y el Propofol es el fármaco hipnótico. Por lo tanto, altos niveles de Remifentanil están asociados con altos niveles de analgesia, los cuales se reflejan en una reducción en la respuesta a estímulos nociceptivos, tal como se observó en el grupo RSS6 (CeRemi = 1,45 0,728 ng/mL). Así mismo, se conoce que el índice BIS es capaz de describir bastante bien el efecto hipnótico, lo cual fue confirmado con los resultados del Análisis 1, pero el BIS no pudo describir correctamente el efecto analgésico y la respuesta a la estimulación nociceptiva. En otros estudios se ha observado que el índice BIS puede presentar un mismo valor para diferentes combinaciones en las concentraciones de Propofol y Remifentanil [22], siendo posible que aunque el BIS presente valores bajos (combinación de mucho Propofol y poco Remifentanil) se pueda observar una respuesta ante la presencia de estímulos dolorosos.

En relación con el análisis de Poincaré, aunque se presentaron diferencias en los valores de $\mathrm{Pk}$ calculados sobre un mismo índice para diferentes valores de retardo, estas diferencias son mínimas cuando se considera el rango de baja frecuencia RF2 (Análisis 2, Tabla 8). Lo anterior está relacionado con el hecho de que las señales consideradas en el Análisis 2 contienen principalmente información de baja frecuencia y por lo tanto el gráfico de Poincaré presentará una forma similar para diferentes tiempos de retardo entre 1 y 8 muestras. Para el Análisis 1, los mejores valores de $\mathrm{Pk}$ se obtuvieron con valores de retardo de 8 muestras en el rango de alta frecuencia RF3, el cual es un tiempo mayor al periodo de las componentes de frecuencia que se encuentran en el rango RF3. Tal como se señaló en las gráficas mostradas en la Figura 5, esta situación sugiere que el gráfico de Poincaré se está construyendo con muestras tomadas en distintos periodos de la señal, indicando que existe una correlación (o anticorrelación) importante entre las muestras de la señal en diferentes periodos de tiempo.

Es importante señalar que en el presente estudio no se aplicaron técnicas avanzadas para el rechazo de artefactos en las señales EEG, lo cual sugiere que el análisis de Poincaré, aunque tuvo buenos resultados, podría mejorar su desempeño con un buen preprocesado que elimine la mayor parte de artefactos en la señal EEG. La eliminación de los puntos identificados como artefactos (datos con 
un voltaje fuera del rango $+/-200 \mu \mathrm{V}$ ) influye muy poco en los resultados y en los análisis realizados considerando que: i) los epochs que se utilizaron son de 10 segundos, las cuales contienen en condiciones ideales 10240 muestras (1024 muestras/s*10 s), permitiendo formar 10239 pares de coordenadas o puntos en el mapa de Poincaré; ii) los índices SD1 y SD2 consideran el efecto conjunto de todos los puntos del epoch y no de una muestra en particular; iii) los análisis se realizaron a partir del valor obtenido en el estadístico $\mathrm{Pk}$, en el cual se compara el comportamiento de los índices SD1 y $\mathrm{SD} 2$ con respecto a las escalas Ramsay en ventanas de 1 minuto. Lo anterior ocasiona que el efecto de una muestra con artefacto se vea enmascarado por todos los demás datos y altere muy poco los resultados obtenidos. Además, el gráfico de Poincaré tiene el potencial de ser una técnica adecuada para analizar la dinámica de señales de corta duración y no requiere que la señal EEG presente características de estacionariedad [11].

\section{CONCLUSIONES}

En el presente trabajo se utilizó el gráfico de Poincaré, el cual es una técnica no lineal, para generar índices que permiten caracterizar los niveles de anestesia ligera a partir de las señales registradas en la frente de los pacientes (EEG+EMG) y de respuestas categóricas observadas, que fueron evaluadas mediante la Escala de Sedación de Ramsay. Estos índices fueron comparados y mezclados con parámetros derivados del análisis espectral de las señales EEG+EMG utilizando modelos ANFIS, con el fin de mejorar el valor de predicción obtenido con los índices de manera individual. Los resultados obtenidos indicaron que los índices de Poincaré calculados sobre las componentes de alta frecuencia de la señal (EMG) contienen información útil relacionada con el nivel de anestesia ligera en un paciente. Particularmente, el índice SD1/SD2, calculado en la banda de frecuencia RF3 $=30-110 \mathrm{~Hz}$ con un retardo de 8 muestras, presentó la mejor predicción del nivel de anestesia ligera, según el valor de la escala de sedación Ramsay. Por otra parte, los índices de Poincaré calculados sobre las componentes de baja frecuencia del EEG mostraron estar más relacionados con la clasificación de las respuestas de los pacientes ante la aplicación de estímulos nociceptivos, siendo los índices SD1 y SD2 los de mejor desempeño (calculados en la banda de frecuencia $\mathrm{RF} 2=0,5-30 \mathrm{~Hz}$ con un retardo de 6 y 2 muestras, respectivamente). Finalmente, una mezcla de parámetros derivados del gráfico de Poincaré y del análisis espectral, combinados con la concentración de los fármacos, permitió obtener niveles de predicción Pk del orden de 0,799. Se puede concluir que los modelos que incluyen los parámetros del gráfico de Poincaré emergen como un buen estimador de los niveles de anestesia ligera.

\section{AGRADECIMIENTOS}

Investigación financiada en parte por el gobierno de Colombia a través de COLCIENCIAS (1217$669-45428$ y 1232-807-64083) y por el gobierno de España a través de MINECO (TEC2013-44666-R y TEC2014-603337-R), el CIBER-BBN, Beca Fin de Residencia del Hospital CLINIC de Barcelona y el FIS (PI/050072 y PS09/01209).

\section{REFERENCIAS}

[1] American Society of Anesthesiologists Task Force on Sedation and Analgesia by Non-Anesthesiologists. "Practice guidelines for sedation and analgesia by non-anesthesiologists. Anesthesiology". Vol. 96, pp. 1004-1017. 2002.

[2] I.J. Rampil. "A primer for EEG signal processing in anesthesia". Anesthesiology. Vol. 89, pp. 980-1002. 1998.

[3] H. Viertiö-Oja, V. Maja, M. Särkelä, P. Talja, N. Tenkanen, H. Tolvanen-Laakso, M. Paloheimo, A. Vakkuri, A. Yli-Hankala and P. Meriläinen. "Description of the Entropy algorithm as applied in the Datex-Ohmeda S/5 Entropy Module". Acta Anaesthesiol. Scand. Vol. 48, pp. 154-161. 2004.

[4] S. Pilge, J. Blum, E.F. Kochs, S. A. Schöniger, M. Kreuzer and G. Schneider. "Does the Cerebral State Index Separate Consciousness from Unconsciousness?". Anesthesia \& Analgesia. Vol. 113, pp. 1403-1410. 2011.

[5] G.A. Mashour, B.A. Orser and M.S. Avidan. "Intraoperative awareness: neurobiology to clinical practice". Anesthesiology. Vol. 114, pp. 1218-1233. 2011.

[6] J.J. Pandit and T.M. Cook. "National Institute for Clinical Excellence guidance on measuring depth of anaesthesia: limitations of EEG-based technology". 
British Journal of Anaesthesia. Vol. 112, pp. 385-386. 2014.

[7] C.J. Chisholm, J. Zurica, D. Mironov, R. R. Sciacca, E. Ornstein and E. J. Heyer. "Comparison of electrophysiologic monitors with clinical assessment of level of sedation". Mayo Clinic proceedings. Vol. 81, pp. 46-52. 2006.

[8] U. Melia, M. Vallverdu, M. Jospin, E.W. Jensen, J.F. Valencia, F. Claria, P. Ambus and P. Caminal. "Prediction of nociceptive responses during sedation by time-frequency representation". IEEE-EMBS Proc. Annual International Conference of the IEEE, pp. 2547-2550. 2013.

[9] U. Melia, M. Vallverdú, X. Borrat, J.F. Valencia, M. Jospin, E.W. Jensen, P. Gambus and P. Caminal. "Prediction of Nociceptive Responses during Sedation by Linear and Non-Linear Measures of EEG Signals in High Frequencies". Plos One, pp. 1-21. 2015.

[10] C.K. Karmakar, A.H. Khandoker, J. Gubbi and M. Palaniswami. "Complex correlation measure: a novel descriptor for Poincaré plot". BioMedical Engineering, pp. 1-12. 2009. DOI: $10.1186 / 1475-925 X-8-17$.

[11] K. Hayashi, T. Yamada, T. Sawa. "Comparative study of Poincaré plot analysis using short electroencephalogram signals during anaesthesia with spectral edge frequency 95 and bispectral index". Anaesthesia, pp. 310317. 2015.

[12] A.K. Golińska. "Poincaré Plots in Analysis of Selected Biomedical Signals". Studies in Logic Grammar Rhetoric. Vol. 35, pp. 117 127. 2013.

[13] A. Goshvarpour, S. Rahati, V. Saadatian and M. Morvarid. "Phase space in EEG signals of women refferred to meditation clinic". J. Biomedical Science and Engineering. Vol. 4, pp. 479-482. 2011.

[14] K. Hayashi, N. Mukaia and T. Sawa. "Poincaré analysis of the electroencephalogram during sevoflurane anesthesia". Clinical Neurophysiology, pp. 404-411. 2015.

[15] M.A. Ramsay, T.M. Savege, B.R. Simpson and R. Goodwin. "Controlled sedation with alphaxalone-alphadolone”. Br. Med. J. Vol. 2, pp. 656-659. 1974.

[16] J.D. Bolaños, M. Vallverdú, P. Caminal, D.F. Valencia, X. Borrat, P.L. Gambus and J.F. Valencia. "Assessment of SedationAnalgesia by means of Poincaré Analysis of the Electroencephalogram". 38th Annual International Conference of the IEEE Engineering in Medicine and Biology Society (EMBC'16). Vol. 38, pp. 6425-6428. 2016.

[17] C. González, E.W. Jensen, P.L. Gambús and M. Vallverdú. "M Poincaré plot analysis of cerebral blood flow signals: Feature extraction and classification methods for apnea detection”. PLoS ONE. Vol. 13, pp. e0208642. 2018.

[18] E.O Brigham. "The Fast Fourier Transform and its applications". Prentice-Hall. 1974.

[19] J. Kortelainen and T. Seppanen. "Electroencephalogram-based depth of anaesthesia measurement: Combining opioids with hypnotics". Trends in Anaesthesia and Critical Care, pp. 270-278. 2013.

[20] W.D. Smith, R. Dutton and N.T. Smith. "Measuring the performance of anesthetic depth indicators". Anesthesiology. Vol. 84, pp. 38-51. 1996.

[21] M. Paloheimo. "Quantitative surface electromyography (qEMG): applications in anaesthesiology and critical care". Acta Anaesthesiol Scand Suppl. Vol. 93, pp. 1-83. 1990.

[22] E. Brocas, H. Dupont, C. Paugam-Burtz, F. Servin, J. Mantz and J.M. Desmonts. "Bispectral index variations during tracheal suction in mechanically ventilated critically ill patients: effect of an alfentanil bolus". Intensive Care Med. Vol. 28, pp. 211-213. 2002. 